\title{
Passivity Based Control of the Double Inverted Pendulum Driven by a Linear Induction Motor
}

\author{
Chin-I Huang, and Li-Chen Fu
}

\begin{abstract}
In this paper, we will propose a nonlinear passivity based controller for a double inverted pendulum system, consisting of a two-link pendulum mounted on a pad which is moved by a linear induction motor so that the double pendulum points straight-up. The proposed controller not only can drive the double pendulum from its "natural stable hanging position" to its "unstable upright inverted position" while linear the induction motor's displacement is brought to zero but also can guarantee that the motor with very unique end-effect can achieve servo motion tracking with high performance. Also, computer simulations are provided to demonstrate the effectiveness of the hereby presented controller design.
\end{abstract}

Index Terms - Double Inverted Pendulum, Underactuated Mechanical System, Linear Induction Motor, Passivity Based Control, Adaptive Backstepping Control

\section{INTRODUCTION}

The general double inverted pendulum system is a twolink (two-degree of freedom) manipulator mounted on a cart (one degree of freedom), where both links are not actuated but the cart is actuated. The main goal is to bring up the two-link pendulum from its "natural stable hanging position" to its "unstable upright inverted position" while the cart is brought to the origin. Since if the cart needs to be driven by a rotary actuator and to move translationally, then a gear and a rotary-to-linear converter will be required to fulfill the purpose. But the most obvious advantage of the linear motor is that it has no power loss due to gears and is free of mechanical rotary-to-linear converters to produce linear motion. Hence, in this paper the cart of the system is in particular actuated by a linear induction motor. For the double inverted pendulum, it is a typical underactuated mechanical system. So far, there have been many similar systems proposed in the literature, such as Acrobot[24], Pendubot [18-20], etc.

Many studies have been conducted for controlling nonlinear underactuated mechanical systems [18-20, 23], which can be roughly classified into two groups respectively associated with swing up control and a balance control. For implementation of the first control strategy, researchers use the concept of partial feedback linearization and passivity to design the swing up controller. For implementation of the latter, people consider the linear quadratic regulator (LQR) design or the pole placement technique based on the plant model linearized around the

Chin-I Huang is with the Department of Electrical Engineering Nat ional Taiwan University, Taipei, Taiwan, R. O. C.

Li-Chen Fu is with the Department of Electrical Engineering and Department of Computer Science and Information Engineering, National Taiwan University, Taipei, Taiwan, R. O. C. (elephone: 1886-2-2362. 2209, e-mail: lichen@csie.ntu.edu.tw). desired equilibrium stabilizing the pendulum at the desired position.

On the other hand, linear induction motors (LIMs) widely used in many industrial applications including transportation, conveyor systems, actuators, material handling, pumping of liquid metal, and sliding door closers, etc. with satisfactory performance. Besides LIMs, linear electric motors also include linear D.C. motors, linear synchronous motors and linear stepping motors. Among these motors, the LIM has many advantages such as simple replacement about the motion device of the motor, reduction of mechanical power losses and of the size of the motion device, silence, high starting thrust force, and easy maintenance, repairing and replacement.

In the early researches, Yamamura has first discovered a particular phenomenon of the end-effect on LIM [1]. A control method, decoupling the control of thrust and the attractive force of a LIM using a space vector control inverter, was presented in R], i.e. by selecting voltage vectors of PWM inverters appropriately.

Although the parameters of the simplified equivalent circuit model of an LIM can be measured by conventional methods (no-load and locked secondary tests), due to limited length of the machine the realization of the no-load test is almost impossible. Thus, the applicability of conventional methods for calculating the parameters of the equivalent model is limited. In order to measure the parameters, application of the finite element (FE) method for determining the parameters of a two-axis model of a three-phase linear induction motor has been proposed in [3]. Another method is proposed by removing the secondary [4].

To resolve the unique end-effect problem for an LIM, speed dependent scaling factors are introduced to the magnetizing inductance and series resistance in the d-axis equivalent circuit of the rotary induction motor (RIM) [5] to correct the deviation caused by the "end-effect". On the other hand, there is a thrust correction coefficient introduced by $[6,7]$ to calculate an actual thrust to compensate for the end effect. A related method to deal with the problem is that an external force corresponding to the end effect is introduced into the RIM model to provide a more accurate modeling of an LIM under consideration of end effect as shown in [8].

The paper is organized as follows. In section 2, we introduce the system model and state the control objectives. Section 3 will develop a passivity-based nonlinear control of the double inverted pendulum to solve the task of bringing up to pendulum from its "natural stable hanging position" to its "unstable upright inverted position". In Section 4, we propose an adaptive backstepping approach to fulfill the tracking linear induction motor. To 
demonstrate the effectiveness of the hereby developed controllers, Section 5 will provide extensive computer simulations with satisfactory performance. Finally, Section 6 gives some concluding remarks.

\section{PROBLEM FORMULATION}

The system of double inverted pendulum consists of a two-linked pendulum mounted on a cart actuated by a linear induction motor to move translationlly along a horizontal track so as to maintain two-link inverted pendulums on the cart. Each link is free to rotate on the vertical plane about an axis associated with an articulated joint, defining the two DOFs, $\theta_{1}(t)$ and $\theta_{2}(t)$. In our setup, we assume that no torque can be around the axis of $\theta_{1}(t)$ and $\theta_{2}(t)$, which are two passive joints. The linear induction motor is an actuator, which exerts a force along the translational DOF $x$. Figure 1 illustrates the system of double inverted pendulum on a cart driven by a linear induction motor. The swing up problem consists in driving the double pendulum from its. "natural stable hanging position" to its "unstable upright inverted position" while the linear induction motor displacement is brought to zero.

In this paper, $=$ we not only consider the pendulum dynamics but also consider the dynamics of the actuator, i.e. linear induction motor. The mathematical model of the double inverted pendulum is constructed using EulerLagrange method under the following assumptions to simplify the analysis:

(A.I) The links of the pendulum are rigid bodies;

(A.2) The inherent friction of the pendulum can be neglected; (A.3) Three phases of the LIM are balanced;

(A.4) The magnetic circuit of the LIM is unsaturated;

(A.5) The LIM is without end 'effect (we will relax this assumption later in the stage of controller design),

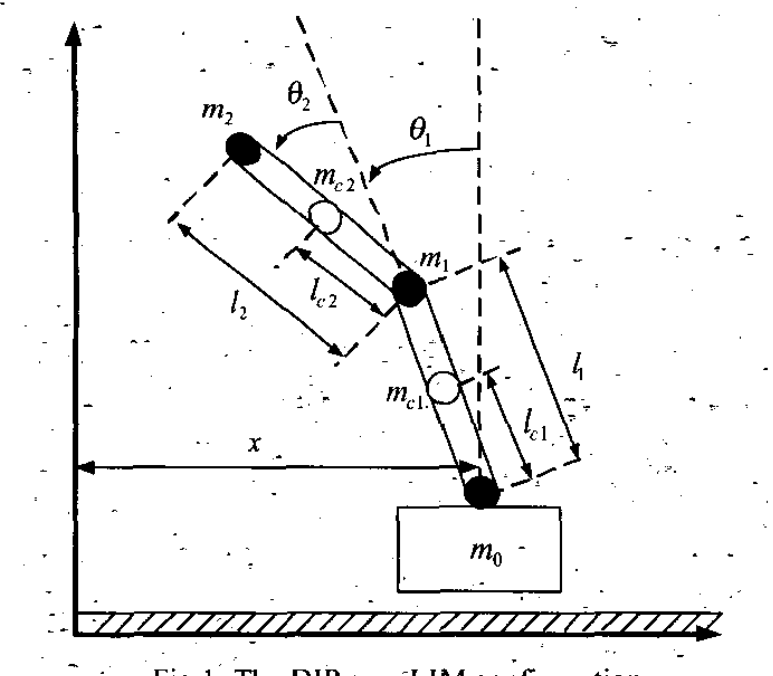

- Fig 1: The DIP on a LIM configuration

- the dynamics of the entire system can be rearranged into. the following more compact form

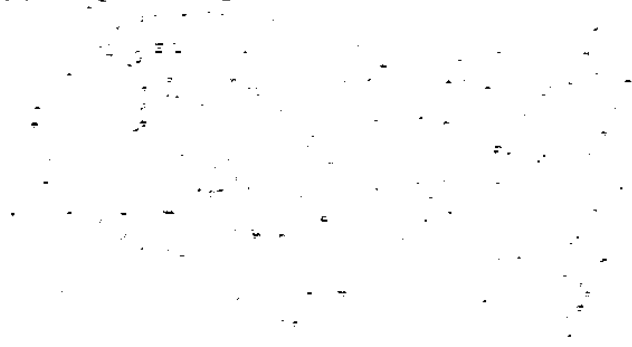

$$
\begin{aligned}
& \dot{i}_{q}=-a_{1} i_{q}+a_{2} \lambda_{q}-\beta p v_{r} \lambda_{d}+c V_{q s} \\
& \dot{i}_{d}=-a_{1} i_{d}+\beta p v_{r} \lambda_{q}+a_{2} \lambda_{d}+c V_{d s} \\
& \dot{\lambda}_{q}=a_{3} i_{q}-a_{4} \lambda_{q}+p v_{r} \lambda_{d} \\
& \lambda_{d}=a_{3} i_{d}-a_{4} \lambda_{d}-p v_{r} \lambda_{q} \\
& F_{e}=K_{f}\left(\lambda_{d} i_{q}-\lambda_{q} i_{d}\right) \\
& F=M(q) \ddot{q}+C(q, \dot{q})+G(q)
\end{aligned}
$$

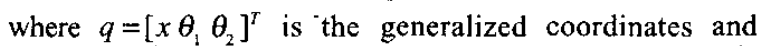
$F=\left[F_{e} 00\right]^{T}$ is vector of the external force. The notations of the inertia matrix $M$, the Coriolis $C$, and gravity $G$, and the parameters involved are shown in Table 1.

\section{PASSIVE SYSTEM AND PASSIVITY BASED CONTROL}

In this section, we try to design the controller which achieves the goal of moving the double pendulum from its "natural stable hanging position" to its "unstable upright inverted position" while the cart driven by the LIM is brought to origin. To facilitate the controller design, we briefly review some of the basic definitions, and the theorem of passivity theory [25].

$$
\begin{aligned}
& \text { 3.1 Basic concepts and definitions of Passive System } \\
& \text { Consider an MIMO nonlinear system of the form } \\
& \qquad \begin{array}{l}
\dot{x}=f(x)+g(x) u \\
y=h(x)
\end{array}
\end{aligned}
$$

where $x \in R^{n}$ is the state, $u \in R^{m}$ is the input, $y \in R^{m}$ is the output, $f$ and $g$ are $C^{\infty}$ vector fields, and $h$ is a smooth mapping (i.e. $C^{\infty}$ ). It is assumed that the vector field $f$ has at least one equilibrium and without loss of generality we asšume that $f(0)=0$ and $h(0)=0$. Let $U$ be the set of admissible inputs that consisting of all reat valued piecewise continuous function defined on $R^{m}$. It is also assumed that for any $u \in U$ and for any $x^{0}=x(0) \in R^{n}$, the output $y(t)=h\left(\phi\left(t, x^{0}, u\right)\right.$ ) of system (2), where $\phi\left(t, x^{0}, u\right)$ denotes the flow of $f(x)+g(x) u$ for any initial condition $x^{0} \in R^{n}$ and for any $u \in U$, is such that $\int_{0}^{t}\left|y^{T}(s) u\right| d s<\infty$, for âil $t \geq 0$, i.e. the energy stored in system (2) is bounded above. :

Definition 1[25]: A system (2) is said to be $C^{r}$ passive if there exists a $C^{r}$ nonnegative function $V: R^{n} \rightarrow R$, called the storage function, which satisfies $V(0)=0$, such that for all $u \in U, x^{0} \in R^{n}, t \geq 0^{-}$

$$
\text { - } V(x)-V\left(x^{0}\right) \leq \int_{0}^{t} y^{T}(s) u(s) d s .
$$

Definition 2[25]: $A$ passive system $\Sigma$ with storage function $V$ is said to be lossless if for all $u \in U, x^{0} \in R^{n^{-}}$, $t \geq 0$

$$
{ }^{\circ} V(x)-V\left(x^{0}\right)^{-}=\int_{0}^{t} y^{T}(s) u(s) d s \quad \because
$$

Definition 3[25]: A passive system $\Sigma$ with storage function $V$ is said to be strictly passive if there exists a 
positive definite function $S: R^{n} \rightarrow R$ such that for all $u \in U, x^{0} \in R^{\dot{n}}, t \geq 0$

$$
V(x)-V\left(x^{0}\right)=\int_{0}^{t} y^{T}(s) u(s) d s-\int_{0}^{t} S(x(s)) d s
$$

Theorem 1|25]: Suppose $\Sigma$ is passive with a storage function $V$ which is positive definite with no critical point other than $x=0$ and such that $L_{f} V(x)=0$ (i.e. lossless system) and if $D=\operatorname{span}\left\{a d_{f}^{k} g_{i}: 0 \leq k \leq n-1,1 \leq i \leq m\right\}$ has dimension $n$ at each $x \neq 0$, then the control law

$$
u=-\left(L_{g} V(x)\right)^{T}=-h^{T}(x)
$$

globally asymptotically stabilize the system.

\subsection{Partial Feedback Linearization}

It has been shown that the dynamics of the double inverted pendulum (1) are not feedback linearizable with static state feedback nor through some nonlinear coordinate transformation [20]. The double inverted pendulum system belongs to the class of underactuated mechanical systems, which have fewer control inputs than the degrees of freedom. We first consider a general underactuated mechanical system with $n$ generalized coordinates and $\mathrm{m}$ actuators. By partitioning the vector $q$, we get $q=\left[q_{\theta}^{T}, q_{x}^{T}\right]$, with $q_{\theta}$ corresponding to the passive variables and $q_{x}$ corresponding to the actuated variables. The dynamics of $n$ degrees of freedom of the general underactuated mechanical system with $q_{\theta}$ passive coordinates and $q_{x}$ actuated coordinates can be written in the following general form [20]:

$$
\begin{aligned}
& M_{\theta \theta} \ddot{q}_{\theta}+M_{\theta x} \ddot{q}_{x}+C_{\theta}(q, \dot{q})+g_{\theta}(q)=0 \\
& M_{x \theta} \ddot{q}_{\theta}+M_{x x} \ddot{q}_{x}+C_{x}(q, \dot{q})+g_{x}(q)=f_{x}
\end{aligned}
$$

Here, $M(q)$ is the symmetric, positive definite inertia matrix, the $C(q, \dot{q})$ includes Coriolis and centrifugal terms, $G$ contains the terms derived from the potential energy, such as gravitational and elastic generalized forces, and $f_{x}$ represents the input of the generalized forces produced by the $m$ actuators at $q_{x}$. In the underactuated mechanical system (7), we first assume that all the parameters are known. Thus we may achieve a linear response from either link, but not both, by applying suitable nonlinear partial feedback linearization. Considering the first formula in (7), one gets

$$
\ddot{q}_{\theta}=-M_{\theta \theta}^{-1}\left(M_{\theta x} \ddot{q}_{x}+C_{\theta}+g_{\theta}\right)
$$

By substituting (8) into the second formula in (7), one obtains

$$
\bar{M}_{x x} \ddot{q}_{x}+\vec{C}_{x}+\bar{g}_{x}=f_{x}
$$

where

$$
\begin{aligned}
& \bar{M}_{x x}=M_{x x}-M_{x \theta} M_{\theta \theta}^{-1} M_{\theta x} \\
& \bar{C}_{x}=C_{x}-M_{x \theta} M_{\theta \theta}^{-1} C_{\theta} \\
& \bar{g}_{x}=g_{x}-M_{x \theta} M_{\theta \theta}^{-1} g_{\theta}
\end{aligned}
$$

Hence, a partial feedback linearizing controller can be defined according to equations (9) and (10) yielding

$$
f_{x}=\bar{M}_{x x} u+\bar{C}_{x}+\bar{g}_{x}
$$

where $u$ is regarded as a new control input. As a result, the complete system can be rewritten as

$$
\begin{aligned}
M_{\theta} \ddot{q}_{\theta}+C_{\theta}+g_{\theta} & =-M_{\theta x} u \\
\ddot{q}_{x} & =u
\end{aligned}
$$

Let the control input $u$ be designed into two parts $u=u_{x}+u_{\theta}$. Then, $u_{x}$ and $u_{\theta}$ are designed such that $q_{x}$. and $q_{\theta}$ will converge to zero as $t \rightarrow \infty$, respectively.

\subsection{Passivity. Based Controller Design}

The partial feedback linearization approach transfers the original system (7) into a simpler one (12) both in concept and in structure. For the reason of convenience, we rewrite system (12) in a more general form

$$
\begin{aligned}
& \dot{\theta}=f(\theta)+g(\theta) u \\
& \dot{x}=A x+B u
\end{aligned}
$$

According to nonlinear $\theta$-subsystem in (13), there exists the total energy $V_{\theta}(\theta)$. The time derivative of the total energy $V(\theta)$ can be derived as

$$
\begin{aligned}
\dot{V}_{\theta}(\theta) & =\frac{\partial V_{\theta}(\theta)}{\partial \theta} \dot{\theta}=\frac{\partial V_{\theta}(\theta)}{\partial \theta}(f(\theta)+g(\theta) u) \\
& =L_{f} V_{\theta}+L_{g} V_{\theta} u
\end{aligned}
$$

By the assumption A.2, the double inverted pendulum system is lossless system. Thus, we have

$$
L_{f} V_{\theta}=0
$$

According to Theorem 1, if the control input is chosen as

$$
u=-\left(L_{g} V_{\theta}\right)^{T}
$$

then the $\theta$ subsystem is globally asymptotically stable. On the other hand, the $x$-subsystem is globally asymptotically stable if the control input $\mathrm{u}$ is selected as $u=-K_{x} x$ such that the transfer function $K_{x}(s I-A)^{-1} B$ is strictly positive real. From the Passivity Theorem [22, 27], we know that the whole system can be rendered passive with the combined control input

$$
u=-\left(L_{g} V_{\theta}\right)^{T}-K_{x} x
$$

Remark: In order to achieve the former control objective, i.e. to stabilize the double inverted pendulum in the "unstable upright inverted position", the following two conditions should be satisfied.

A) $x=0, \dot{x}=0$;

B) $V_{\theta}=V_{u p}=g\left(2 m_{c 2} l_{c 2}+2 m_{2} l_{2}+m_{1} l_{1}+m_{2} l_{1}+m_{c 2} l_{1}+m_{c 1} l_{c 1}\right)$;

where the second condition refers to the double inverted pendulum at the desired unstable upright inverted position with zero linear displacement and zero linear velocity. The explicit energy expression of the double inverted pendulum, when under the above conditions, is:

$$
\begin{aligned}
V_{\theta} & =\frac{1}{2} M_{22} \dot{\theta}_{1}^{2}+\frac{1}{2} M_{33}\left(\dot{\theta}_{1}+\dot{\theta}_{2}\right)^{2}+M_{2} \dot{\theta}\left(\dot{\theta}_{1}+\dot{\theta}_{2}\right) \cos \theta_{2} \\
& +g\left(2\left(m_{22} l_{c 2}+m_{2} h_{2}\right) \cos \left(\theta+\theta_{2}\right)+\left(m_{1} l_{1}+m_{21} L_{1}+m_{c 2} l_{1}+m_{2} l_{d}\right) \cos \theta\right) \\
& =V_{t w}
\end{aligned}
$$

The control input which can successfully swing up the double pendulum from its "natural stable hanging position". to its "unstable upright inverted position" is shown to be

$$
u=-k_{p} x-k \dot{x}
$$

$+k_{\theta}\left(V_{\theta}-V_{i p}\right)\left(\left(m_{1} l_{1}+m_{c 2} l_{1}+m_{d} l_{\mathrm{d}}+m_{21}\right) \dot{\theta} \cos \theta_{1}+2\left(m_{2} l_{2}+m_{c 2} l_{c}\right)\left(\dot{\theta}+\dot{\theta}_{2}\right) \cos \left(\theta_{1}+\theta_{2}\right)\right)$

However, he real control input is not "force", but is "voltage" according to the full dynamics of double inverted pendulum system driven by LIM. Since the above proposed passivity based controller can make the system of pendulum globally asymptotically stable, in the following context we will assume that the desired motion trajectory $x_{d}$ of LIM can be sought. Hence, the problem to find the 
force/torque control to swing up the double inverted pendulum problem can be changed as motion tracking problem for an LIM. In the next section, we will propose a position controller to achieve the objective of motion tracking of the LIM. An adaptive position controller is proposed to deal with uncertainty of the mutual inductance.

\section{ADAPTIVE BACKSTEPPING CONTROLLER DESIGN}

The fundamental difference between a rotary induction motor and a LIM is the finite length of the magnetic and electric circuit of the LIM along the direction of the travelling field. The open magnetic circuit causes an initiation of the so-called longitudinal end-effects [5].

For a LIM, the end effect with the load force can be represented as a function of the speed $\dot{x}$, which can be normally simplified into the form

$$
F_{E}=\sum_{n=0}^{2} b_{n} \dot{x}^{n}+M_{e} \ddot{x} \triangleq M_{e} \ddot{x}+b_{o}+b_{i} \dot{x}+b_{2} \dot{x}^{2}
$$

In this paper, the end-effect is assumed in the aforementioned form as $F_{E}=\Theta V_{r}^{T}$ with the unknown constant parameters $\Theta=\left[\begin{array}{llll}M_{e} & b_{0} & b_{1} & b_{2}\end{array}\right]$, and a known function vector $V_{r}^{T}=\left[\begin{array}{llll}\ddot{x} & \dot{x}^{0} & \dot{x}^{1} & \dot{x}^{2}\end{array}\right]$. The joint mass $M=m_{0}+M_{e}$ is therefore also unknown, which leads to the total mechanical load with motor itself as $F_{e}=\Theta^{T} V_{r}$, where $\Theta^{T}=\left[\begin{array}{llll}M & b_{0} & b_{1} & b_{2}\end{array}\right]$. To proceed further, we introduce some more assumption as shown below:

(A.6) $x_{2}=\lambda_{q}^{2}+\lambda_{d}^{2}>0$,

(A.7) The desired speed should be a bounded smooth function with known first and second order time derivatives, then further simplify the dynamics shown in (1) by introducing a nonlinear coord inate transformation given as follows:

$$
\begin{aligned}
& x_{1}=i_{q}^{2}+i_{d}^{2} \\
& x_{2}=\lambda_{q}^{2}+\lambda_{d}^{2} \\
& x_{3}=i_{q} \lambda_{q}+i_{d} \lambda_{d} \\
& x_{4}=i_{q} \lambda_{d}-i_{d} \lambda_{q} \\
& x_{5}=\dot{x}
\end{aligned}
$$

Remarks: The transformation is trying to make the secondary flux norm, the electric force and the rotor speed as individual variables $x_{2}, x_{4}$ and $x_{5}$, respectively, and certainly the nonlinear transformation is not unique. Intially, we adopt the stator voltage inputs as $c V_{d s}=\frac{-\lambda_{q}}{\sqrt{\lambda_{d}^{2}+\lambda_{q}^{2}}} V, c V_{d s}=\frac{\lambda_{d}}{\sqrt{\lambda_{d}^{2}+\lambda_{q}^{2}}} V[17,22]$, with such transformation, then the dynamical equations shown in (1) can thus be transformed into the following dynamic model:

$$
\begin{aligned}
\dot{x}_{1} & =-2 a_{1} x_{1}+2 a_{2} x_{5}+\left(2 x_{4} / \sqrt{x_{2}}\right) V \\
\dot{x}_{2} & =-2 a_{4} x_{2}+2 a_{3} x_{3} \\
\dot{x}_{3} & =a_{3} x_{1}+a_{2} x_{2}-\left(q+a_{4}\right) x_{3}+p x_{5} x_{4} \\
\dot{x}_{4} & =-p x_{5} x_{3}-\beta p x_{5} x_{2}-\left(a+a_{4}\right) x_{4}+\sqrt{x_{2}} V \\
M \dot{x}_{5} & =K_{f} x_{4}-\sum_{n=0}^{2} b_{n} x_{5}^{n}
\end{aligned}
$$

To control the system (19) we develop the position controller to achieve the goal $x_{r} \rightarrow x_{d}$ as introduced in the following section.
Now, we introduce another state

$$
x_{6}=x_{r}
$$

to facilitate investigation of the development of a position controller. Then, define the tracking errors as follows:

$$
e_{p}=x_{r}-x_{d} \triangleq e_{6}
$$

Normally, while the position tracking error is driven to zero, the speed is also regulated to zero. Thus, we naturally define a joint error signal $S$ as follows:

$$
S=\dot{e}_{p}+a e_{p}=e_{5}+a e_{6},
$$

where $a$ is a positive scalar gain, and note the case with $a=0$ will be degenerated back speed tracking problem.

Theorem 2. Consider an LIM whose dynamics are governed by system (19) under the assumptions (A.6). Given a third-time differentiable smooth desired position trajectory $x_{d}$ with $x_{d}, \dot{x}_{d}, \ddot{x}_{d}$ and $\dddot{x}_{d}$ being all bounded, then the following control input can achieve the control objective $x_{r} \rightarrow x_{d}$ (i.e. $x_{6}=x_{r}$ will follow $x_{d}$ asymptotically) with the control input

$$
V_{q s}=\frac{\lambda_{d}}{\sqrt{\lambda_{q}^{2}+\lambda_{d}^{2}}} \frac{V}{c}, V_{d s}=\frac{-\lambda_{q}}{\sqrt{\lambda_{q}^{2}+\lambda_{d}^{2}}} \frac{V}{c},
$$

and

$$
V=\frac{1}{\sqrt{x_{2}}}=\left[g_{2}(x)+\hat{\Theta}_{2} W_{2}-K_{f} z_{1}-\rho_{2} z_{2}\right]
$$

with adaptation law

$$
\dot{\tilde{\Theta}}=\dot{\hat{\Theta}}=-\Gamma_{1} z_{1} W, \dot{\tilde{\Theta}}_{2}=\dot{\hat{\Theta}}_{2}=-\Gamma_{2} z_{2} W_{2},
$$

where $\Gamma_{1}, \Gamma_{2}>0$, and $z_{1}=S, z_{2}=x_{4}-\alpha_{2}$,

$$
\alpha_{2}=-\rho_{1} M S+\frac{1}{K_{f}} \Theta^{T} W-\frac{a}{K_{f}} M e_{5}
$$

for some $\rho_{1}, \rho_{2}>0$, and

$$
\begin{aligned}
& g_{2}(x)=p x_{3} x_{5}+\beta x_{2} x_{5}+\left(q+a_{4}-a\right) x_{4}-\rho_{1}\left(K_{f} x_{4}+\kappa J e_{6}\right) \\
& \Theta_{2}^{T} W_{2}=\left(\rho_{1}+\frac{a}{K_{f}}\right) \Theta^{T} W+\frac{1}{K_{f}} \Theta^{T} \dot{W}=\left(\rho_{1}+\frac{a}{K_{f}}\right) \Theta^{T} W+\frac{1}{K_{f}} \Theta^{\prime} W^{\prime}
\end{aligned}
$$

with the parameter vector $\Theta^{\prime}$ as well as the known function vector $W^{\prime}$ satisfying $\Theta^{T} \dot{W}=\Theta^{\prime T} W^{\prime}$.

Proof:

Step 1. Choose a different stabilizing function $\alpha_{2}$ as follows

$$
\alpha_{2}=-\rho_{1} M S+\frac{1}{K_{f}} \hat{\Theta}^{T} W-\frac{a}{K_{f}} M e_{5}
$$

where $\hat{\Theta}$ denotes the on-line parameter estimate. And, redefine the new error variables $z_{1}=S, \mathrm{z}_{2}=x_{4}-\alpha_{2}$.

Evaluate the time derivative of the Lyapunov function candidate

$$
V_{1}=\frac{1}{2} M z_{1}^{2}+\frac{1}{2 \Gamma_{1}} \tilde{\Theta}^{T} \tilde{\Theta},
$$

along the solution trajectories to obtain

$$
\dot{V}_{1}=-\rho_{1} K_{f} M z_{1}^{2}+K_{f} z_{1} z_{2}+\tilde{\Theta}^{T}\left(\frac{1}{\Gamma_{1}} \dot{\tilde{\Theta}}+z_{1} W\right)
$$

Devise the adaptation law as

$$
\dot{\tilde{\Theta}}=\dot{\hat{\Theta}}=-\Gamma_{1} z_{1} W
$$

for some proper positive adaptation gain $\Gamma_{1}$, then (25) can be slightly simplified as: 


$$
\dot{V}_{1}=-\rho_{1} K_{f} M z_{1}^{2}+K_{f} z_{1} z_{2}
$$

Step 2. The time derivative of $z_{2}$ is now expressed as

$$
\dot{z}_{2}=\dot{x}_{4}-\dot{\alpha}_{2}=-g_{1}(x)-\Theta_{1}^{T} W_{1}+\sqrt{x_{2}} V
$$

where the function are as previously defined. Thus, we need to select a Lyapunov function candidate and design $V$ to render its time derivative nonpositive. We want to apply the augmented Lyapunov function candidate as:

$$
V_{2}=V_{1}+\frac{1}{2} z_{2}^{2}
$$

whose time derivative is found to be

$$
\dot{V}_{2}=-\rho_{1} K_{f} M z_{2}^{2}+K_{f} z_{1} z_{2}+z_{2}\left[-g_{1}(x)-\Theta W_{1}+\sqrt{x_{2}} V\right] \text {, }
$$

The control law $V$ should be able to cancel the indefinite term in (17). On the other hand, to deal with the unknown parameters $\Theta_{2}$, we will try to employ the current estimates $\hat{\Theta}_{1}$, i.e.,

$$
V=\frac{1}{\sqrt{x_{2}}}\left[g_{1}(x)+\hat{\Theta}_{1} W_{1}-K_{f} z_{1}-\rho_{2} z_{2}\right]
$$

From this resulting derivative

$$
\dot{V}_{2}=-\rho_{1} K_{f} M z_{1}^{2}+z_{2} \hat{\Theta}_{1} W_{1}-\rho_{2} z_{2}^{2}
$$

in order to cancel the last term in (32), we modify the Lyapunov function as below:

$$
V_{3}=V_{2}+\frac{1}{2} z_{2}^{2}+\frac{1}{2} \tilde{\Theta}_{1}^{T} \tilde{\Theta}_{1},
$$

and the time derivative of $V_{3}$ hence is

$$
\dot{V}_{3}=-\rho_{1} K_{f} M z_{1}^{2}+\hat{\Theta}_{1}^{T}\left(z_{2} W_{2}+\frac{1}{\Gamma_{2}} \dot{\tilde{\Theta}}_{1}\right)
$$

Now, the term with $\tilde{\Theta}_{3}$ can be eliminated completely with the update law

$$
\dot{\tilde{\Theta}}_{2}=\dot{\hat{\Theta}}_{2}=-\Gamma_{2} z_{2} W_{2}
$$

for some positive adaptation gain $\Gamma_{2}$, which thus yields

$$
\dot{V}_{3}=-\rho_{1} K_{f} M z_{1}^{2}-\rho_{2} z_{2}^{2}
$$

which guarantees boundedness of all parameter estimates $\bar{\theta}, \hat{\theta}_{1}$ and $z_{1}, z_{2}$, and $z_{1} \in L^{2} \cap L^{\infty}$. To show boundedness of the rest of states, we can rearrange the dynamical equations from system (19) as shown below:

$$
\dot{X}=\left[\begin{array}{l}
\dot{x}_{1} \\
\dot{x}_{2} \\
\dot{x}_{3}
\end{array}\right]=\left[\begin{array}{ccc}
-2 a_{1} & 0 & 2 a_{2} \\
0 & -2 a_{4} & 2 a_{3} \\
a_{3} & a_{2} & -\left(a_{1}+a_{4}\right)
\end{array}\right]\left[\begin{array}{l}
x_{1} \\
x_{2} \\
x_{3}
\end{array}\right]+\left[\begin{array}{c}
\frac{2 x_{4}}{\sqrt{x_{2}}} V \\
0 \\
p x_{5} x_{4}
\end{array}\right],
$$$$
=A X+u
$$

where A can be shown to be Hurwitz. After reviewing definitions of $x_{3}$ and $\mathrm{V}$, repectively, we found that the first entry of $\mathrm{u}$ will be bounded because $x_{2}$ grows no slower than $x_{3}$ if $x_{3}$ does grow unbounded (due to the second equation of (19)). As a result, $u$ is apparently bounded, and hence $X$ will be bounded. This then proves the boundedness of all the states. We note that $\dot{z}_{1}$ is also bounded, and hence by Barbalat's lemma we can conclude $\dot{z}_{1} \in L_{\infty}$ so that

$$
\lim _{t \rightarrow \infty} z_{1} \rightarrow 0 \text {, i.e., } x_{r} \rightarrow x_{d} \text { as } t \rightarrow \infty \text {. }
$$

\section{SIMULATION RESULTS}

In our simulation, the parameters of the linear induction motor we use are according to Table 2 . All the results will be demonstrated in the following sections.

The two-link pendulum start form its "natural stable hanging position" $\left(\theta_{1}=\pi, \theta_{2}=0\right)$ to its "unstable upright inverted position" ( $\left.\theta_{1}=0, \theta_{2}=0\right)$ while the LIM is brought to the origin.

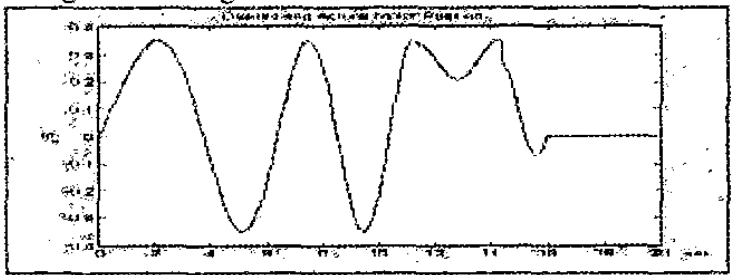

Fig. 2 Desired and Actural Motor Position $x$

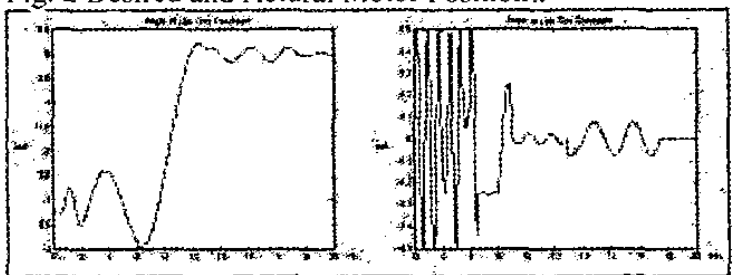

Fig. 3 Angle of Link One $\theta_{1}$ Fig 4. Angle of link Two $\theta_{2}$

\section{CONCLUSION}

In this paper, we have proposed a nonlinear passivity controller and an adaptive backstepping controller for double inverted pendulum mounted on a linear induction motor. The controller which not only swing up but also balance the two linked inverted pendulums on the motor. Stability analysis based on Lyapunov theory is performed to guarantee the controller design is stable. Finally, the simulation results confirm the effectiveness of our control design.

\section{REFERENCES}

[1] Yamamura, S. , Theory of Linear Induction Motors, John Wiley \& Sons, 1972.

[2] Takahashi, 1. and Y. Ide, "Decoupling Control of thrust and attractive force of a LIM using a space vector control inverter", IEEE Trans on Industry Applications, $\mathrm{V}_{\mathrm{ol}} .29, \mathrm{pp}$. $161-167,1993$

[3] Dolinar, D., G. Stumberger and B. Grcar, "Calculation of the Linear Induction Motor Model Parameters using Finite Elements", IEEE Trans. on Mágnetics, Vol. 34, No.5, pp.3640-3643, 1998.

[4] Zhang, Z., Tony R. Eastham and G. E. Dawson "LIM Dynamic Performance Assessment from Parameter Identification", Porc. of Industry Application Society Annual Meeting, Vol. 2, pp. 1047-1051, 1993.

[5] Sung, J. H. and K. Nam, "A New Approach to Vector Control for a Linear Induction Motor considering End Effects," Industry Application Conference, pp. 2284-2289, 1999.

[6] Lee, J. H., S. C. Ahn and D. S. Hyun "Dynamic Characteristic Analysis of Vector Controlled LIM by Finite Element Method and Expcriment", Proc. of Industry Applications Conference, pp.799-806, 1998.

[7] Kwon, B., K. Woo, S. Kim " Finite Element Analysis of Direct Thrust-controlled Linear induction motor", pp, 13061309, IEEE Trans. on Magnetics, Vol. 35, No.3, 1999.

[8] Creppe, R.C. etc., "Dynamic Behavior of a Linear Induction Motor", Proc. of Meditterranean Electrotechnical Conference, 
Vo. 2, 1998.

[9] Shanmugasundaram, A. and M. Rangasamy, "Control of compensation in linear induction motors", IEE proceedings, Vol. 135, pp.22-32, 1988.

[10] Lin, F. J. and C. C. Lee, “ Adaptive Backstepping Control for Linear Induction Motor Drive to Track Periodic Reference", IEE Proc. Electr. Power Appl. Vol. 147, No. 6, pp.449-458, 2000.

[11] Gastli, A., "Compensation for the Effect of Joints in the Secondary Conductor of a Linear Induction Motor", IEEE Trans. on Energy Conversion, Vol. 13, No. 2, pp.111-116, 1998.

[12] Groot, D. J., "Dimensional Analysis of the Linear induction Motor", IEE Proceeding- $B$, Vol. 140, pp. 273-280, 1993.

[13] Nasar, S. A. and I. Boldea, Linear Motion Electric Machines, John Wiley \& Sons, 1976.

[14] Gieras, J. F., Linear Induction Drives, Oxford University Press, 1994.

[15] Krause, P. C., Analysis of Electric Machinery, McGrraw-Hill, 1986

[16] Sastry, S. and M. Bodon, Adaptive control: Stability, Convergence, and Robustness, Englewood cliffs, NJ: Prentice-Hall,1989

[17] Lee, H. T., L. C. Fü and H. S. Huang, " Speed Tracking Control with Maximal Power Transfer of Induction Motor", Proc. IEEE $39^{\text {th }}$ Conf. Decision and Control, pp925-930, 2000.

[18] Spong M. W.. Energy based control of a class of underactuated mechnical systems. Proc. of 1996 IFAC World Congress, Samn Francisco,CA, July, 1996.

[19] Spong M. W.. Underactuated mechanicak systems. In Siciliano B., Valavanis .P. (Eds.), Control Problems in Robotics and Automation,Lecture notes in Control and Information Sciences 230,Springer-Verlag, London, UK, 1997.

[20] Spong M. W., Praly L.Control of underactuated mechanical systems using switching and saturation. Proc. of the Block Island Workshop on Control Using Logic Based Switching, Springer-Verlag, London, UK, 1996.

[21] Sepulchre R., Jankovic M., Kokotovic P. Constructive Nonlinear Control. Springer, London, 1997.

[22] Lee, H. T., L. C. Fu and H. S. Huang, "Sensorless Speed Trackingof Induction Motor with Unknown Torque Based on Maximal PowerTransfer ", IEEE Trans. Ind. Electron., vol. 49 , no. 4, p.p. $911-924,2002$.

[23] K. J. Astrom and K.Furuta. "Swing Up a Pendulum by Energy Control," Automatica, vol. 36. pp. 287-295, 2000.

[24] M. W Spong, "The Swing Up Control Problem for the Acrobot," IEEE Contr. Syst. Mag., Vol. 15. pp. 49-55,1995

[25] C. I. Byrenes, A. Isidori, and J. C. Willems, "Passivity, Feedback Equivalence, and the Global Stablization of Minimum Phase Nonlinear Systems", IEEE Tans. Auto. Cont. vol. 36, pp. 1228-1240, 1991 .

[26] Zhong, W and H. Rock, "Energy and Passivity Based Control of the Double Inverted Pendulum on a Cart", Proc. IEEE Conf. on Control Application, 2001

Table 2: Specification and parameter of the motor

\begin{tabular}{|l|l|}
\hline \multicolumn{1}{|c|}{ Specification } & \multicolumn{1}{c|}{$\begin{array}{c}\text { Parameters normal } \\
\text { value }\end{array}$} \\
\hline 3 Phase (Y-connected) & $\mathrm{R}_{\mathrm{s}}=13.2 \Omega$ \\
Rated Power 1HP & $\mathrm{R}_{\mathrm{r}}=11.78 \Omega$ \\
Rated Air gap $\quad 0.125 \mathrm{in}$ & $\mathrm{L}_{\mathrm{s}}=0.42 \mathrm{H}$ \\
Rated Current $5 \mathrm{~A}$ & $\mathrm{~L}_{\mathrm{r}}=0.42 \mathrm{H}$ \\
Rated Voltage 240V $\quad 4$ & $\mathrm{~L}_{\mathrm{m}}=0.4 \mathrm{H}$ \\
Rated Poles 46.5mm & $\mathrm{M}=4.775 \mathrm{Kg}$ \\
Pitch $\quad \mathrm{B}=53 \mathrm{Kg} / \mathrm{sec}$ \\
Secondary length & $l_{1}=0.39 \mathrm{~m}$ \\
$m_{0}=4.775 \mathrm{~kg}$ & \\
$m_{1}=0.12 \mathrm{~kg}$ & \\
\hline
\end{tabular}

\begin{tabular}{|l|l|}
\hline$m_{2}=0.02 \mathrm{~kg}$ & $l_{2}=0.395 \mathrm{~m}$ \\
$m_{c 1}=0.08 \mathrm{~kg}$ & $l_{c 1}=0.195$ \\
$m_{\mathrm{c} 2}=0.08 \mathrm{~kg}$ & $l_{\mathrm{c} 2}=0.1975 \mathrm{~m}$ \\
& $I_{i}=m_{c} l_{i}^{2} / 12$ \\
\hline
\end{tabular}

Table 1: Nomenclature

$\begin{array}{ll}m_{o}, m_{1}, m_{2} & \text { Mass of LIM and joints } \\ m_{\mathrm{c} 1}, m_{\mathrm{c} 2} & \text { Mass of each pendulum } \\ l_{1}, l_{2} & \text { Length of each pendulum } \\ I_{1}, I_{2} & \text { Moment of inertia of each pendulum } \\ l_{c 1}, l_{c 2} & \text { Length of each pendulum from axis to centre }\end{array}$

$l_{c 1}, l_{c 2}$ Length of each pendulum from axis to centre of mass

$V_{\mathrm{qs}}\left(V_{d s}\right) \quad \mathrm{q}-(\mathrm{d}-)$ Axis input stator voltage

$i_{q}\left(i_{d}\right) \quad \mathrm{q}-(\mathrm{d}-)$ Axis input stator current

$R_{s}\left(R_{r}\right) \quad$ Primary (secondary) resistance

$L_{s}\left(L_{r}\right) \quad$ Primary (secondary) inductance

$\lambda_{q}\left(\lambda_{c r}\right) \quad q-(d-)$ Axis rotor flux

$v_{r} \quad$ Linear speed of the primary

$p_{r} \quad$ Position of the primary

$M_{m} \quad$ Primary mass

$B \quad$ Viscous friction coefficient

$F_{e} \quad$ Electromagnetic force

$F_{L}^{\prime} \quad$ Mechanical load force

$L_{m} \quad$ Mutual inductance

$P \quad$ Number of pole pairs

$\tau \quad$ Pole pitch

$K_{f} \quad$ Force constant $\left(=3 P L_{m} \pi / 2 \tau L_{r}\right)$

$a_{2}=\beta R_{r} / L_{r}, a_{3}=L_{m} R_{r} / L_{r}, a_{4}=R_{r} / L_{r}, D=L_{s} L_{r}-L_{m}^{2}$,

$p=P \pi / \tau, \beta=L_{n} / D, a_{1}=\left(R_{r} L_{r}\right) / D+\beta L_{m} R_{r} / L_{r}$

$M(q)=\left[\begin{array}{lll}M_{11} & M_{12} & M_{\mathfrak{B}} \\ M_{21} & M_{22} & M_{23} \\ M_{31} & M_{32} & M_{33}\end{array}\right] q(q, \dot{q})=\left[\begin{array}{lll}C_{11} & C_{12} & C_{13} \\ C_{2} & C_{22} & C_{23} \\ C_{31} & C_{32} & C_{33}\end{array}\right]$

$G(q)=\left[\begin{array}{l}G_{1} \\ G_{2} \\ G_{3}\end{array}\right], q=\left[\begin{array}{l}x \\ \theta_{1} \\ \theta_{2}\end{array}\right], F=\left[\begin{array}{c}F_{e} \\ 0 \\ 0\end{array}\right]$

$M_{11}=m_{0}+m_{1}+m_{1}+m_{2}+m_{2}$

$M_{22}=m_{1} l_{c 1}^{2}+I_{1}+\left(m_{1}+m_{2}+m_{2}\right) l_{1}^{2}+2\left(m_{22} l_{c 2}+m_{2} l_{2}\right)_{1} \cos \theta_{2}+m_{22} l_{c 2}^{2}+I_{2}+m_{2} l_{2}^{2}$

$M_{33}=m_{22} l_{22}^{2}+I_{2}+m_{2} l_{2}^{2}$

$M_{\mathrm{L}}=M_{\mathrm{z}}=-\left(m_{\mathrm{c} 1 d} l_{d}+m_{1} l_{1}+m_{c 2} l_{1}+m_{21} l_{1}\right) \cos \theta-\left(m_{22} l_{c 2}+m_{2} l_{2}\right) \cos \left(\theta_{1}+\theta_{2}\right)$

$M_{\mathfrak{B}}=M_{31}=-\left(m_{t 2} l_{c 2}+m_{2} h\right) \cos \left(\theta+\theta_{2}\right)$

$M_{32}=M_{32}=\left(m_{22} l_{c 2}+m_{2} l_{2}\right) l_{1} \cos \theta_{2}+m_{22} l_{c 2}^{2}+I_{2}+m_{2} l_{2}^{2}$

$C_{11}=C_{2}=C_{31}=C_{33}=0$

$C_{\mathrm{n}}=\left(m_{1} l_{1}+m_{2} h_{1}+m_{s} l_{c}+m_{21}\right) \dot{\theta} \sin \theta_{1}+\left(m_{2} l_{2}+m_{22} l_{c 2}\right)\left(\dot{\theta}_{1}+\dot{\theta}_{2}\right) \sin \left(\theta+\theta_{2}\right)$

$C_{\mathrm{B}}=\left(m_{2} l_{2}+m_{2} l_{2}\right)\left(\dot{\theta}_{1}+\dot{\theta}_{2}\right) \sin \left(\theta+\theta_{2}\right)$

$C_{22}=\left(m_{2} l_{2}+m_{2} l_{c 2}\right) l_{1}\left(\dot{\theta}-\dot{\theta}_{2}\right) \sin \theta_{2}$

$C_{\mathrm{B}}=-\left(m_{2} l_{2}+m_{2} l_{c 2}\right) l_{1}\left(\dot{\theta}+\dot{\theta}_{2}\right) \sin \theta_{2}$

$C_{x}=\left(m_{2} l_{2}+m_{2} l_{c 2}\right) l_{1} \dot{\theta} \sin \theta_{2}$

$G=0$

$G_{2}=g\left(\left(m_{c 2} l_{c 2}+m_{2} l_{2}\right) \cos \left(\theta+\theta_{2}\right)+\left(m_{1} l_{1}+m_{21} l_{1}+m_{22} l_{1}+m_{d_{1}} l_{1}\right) \cos \theta\right)$

$G=g\left(m_{r 2 c 2} l_{c 2}+m_{22}\right) \cos \left(\theta_{1}+\theta_{2}\right)$ 